\title{
Length-dependent changes of lower limb muscle morphology in Chronic Inflammatory Demyelinating Polyneuropathy assessed with magnetic resonance imaging
}

\author{
Jacob Fanous (1), Alexander M. Zero (1), Kevin J. Gilmore (3), Timothy J. Doherty \\ (4,5), Charles L. Rice $(1,2)$
}

(1) School of Kinesiology, The University of Western Ontario, London, ON, Canada; (2) Department of Anatomy and Cell Biology, Schulich School of Medicine and Dentistry, The University of Western Ontario, London, ON, Canada; (3) Department of Surgery, University of Toronto, Toronto, ON, Canada; (4) Department of Clinical Neurological Sciences, Schulich School of Medicine and Dentistry, The University of Western Ontario, London, ON, Canada ; (5) Department of Physical Medicine and Rehabilitation, Schulich School of Medicine and Dentistry, The University of Western Ontario, London, ON, Canada.

This article is distributed under the terms of the Creative Commons Attribution Noncommercial License (CC BY-NC 4.0) which permits any noncommercial use, distribution, and reproduction in any medium, provided the original author(s) and source are credited.

\begin{abstract}
The objective of the present study was to assess muscle quantity of the thigh and leg in patients with chronic inflammatory demyelinating polyneuropathy (CIDP) compared to age and sex matched controls in exploring length-dependent changes of innervated muscles. In five people with CIDP and seven controls, magnetic resonance imaging was used to assess muscle morphology of the four parts of the quadriceps and medial hamstring muscles. Findings were compared to the triceps surae from a subset of participants. The CIDP group had less contractile tissue in the quadriceps $(11.5 \%, \mathrm{P}<0.05)$, hamstrings $(15.6 \%, \mathrm{P}<0.05)$ and triceps surae $(35.9 \%$, $\mathrm{P}<0.05)$ compared to controls. Additionally, CIDP had less contractile tissue $(18.7 \%)$ in the triceps surae compared to the hamstrings $(\mathrm{P}<0.05)$. Muscle quantity in the quadriceps and hamstrings in CIDP was less than controls, but differences were greater for the distal triceps surae. These findings support a length-dependent affect of CIDP on limb musculature composition.
\end{abstract}

Key Words: Chronic inflammatory demyelinating polyneuropathy; CIDP; muscle quality; muscle composition; MRI.

Eur J Transl Myol 31 (4): 10200, 2021 doi: 10.4081/ejtm.2021.10200

Chronic inflammatory demyelinating polyneuropathy (CIDP) is an autoimmune disease involving multifocal demyelination and secondary axonal degeneration of peripheral nerves and spinal roots. Patients with CIDP present with symmetric sensory and motor deficits in upper and lower limbs, with weakness of both distal and proximal musculature. ${ }^{1}$ Segmental demyelination in CIDP causes the myelin sheath to become discontinuous leading to disturbances in axonal conductance. ${ }^{2}$ Studies have focused on the neuropathic aspects of CIDP and utilized magnetic resonance imaging (MRI) to assess nerve lesions. ${ }^{3,4}$ Lesions in the lumbar (lumbosacral) nerve roots, sciatic nerve, ${ }^{5}$ tibial nerve, and brachial plexus have been shown. ${ }^{6,7}$ The consequences these lesions have on skeletal muscle morphology have been less well described. In addition to assessing total muscle mass in limb muscle groups, MRI is useful to investigate muscle quality by quantifying the amount of noncontractile tissue (such as adipose and connective tissue) infiltration within a muscle compartment. To date, only a few studies have systematically described the impact of nerve lesions on muscle morphology in patients with CIDP. ${ }^{8-10}$ Both studies by Gilmore and colleagues focused on muscles of the leg, finding greater noncontractile tissue volume in those with CIDP compared to age and sex-matched controls for both the tibialis anterior ( $\sim 58 \%)$ and the triceps surae $(\sim 82 \%) .{ }^{9,10}$ Some studies have shown that the impact of CIDP may be length dependent with distal musculature being more severely affected than proximal. ${ }^{1,11-14}$ One reason may be due to the greater immune response in distal nerve terminals, where there is an anatomical deficiency in the blood nerve barrier. $^{15,16}$ Additionally, electrophysiological studies have shown that nerve impairment 
appears to be length dependent with a greater reduction in compound muscle action potential amplitude in distal musculature. ${ }^{11}$ The extent to which this lengthdependence is expressed by differential changes in skeletal muscle quantity, by comparing proximal to distal muscles, has not been explored systematically. The two studies to date that assessed length dependence of muscle composition relied either on semi-quantitative measurements of fatty infiltration using a graded system in computed tomography ${ }^{13}$ or did not follow muscles innervated by the same nerve ${ }^{14}$ to assess more directly a length dependent effect. To investigate length dependent changes in muscle composition, we focused on lower limb musculature innervated by the sciatic nerve, which has long axons suppling major posterior thigh muscles as well as all leg and foot muscles. The sciatic nerve has been shown previously to display noticeable nerve lesions. ${ }^{5}$ Here we studied proximal thigh muscles by quantitatively measuring muscle volumes, using MRI, in the medial posterior thigh compartment of the hamstrings (semimembranosus (SM) and semitendinosus (ST)). Results for the triceps surae were derived from a subset of the same participants included in a previous study on both structure and function of the triceps surae. ${ }^{10}$ In addition, and although not innervated by the sciatic nerve, to provide more comprehensive insight into the thigh, all muscles of the anterior thigh compartment (quadriceps femoris - femoral nerve), including the rectus femoris (RF), vastus intermedius (VI), vastus medialis (VM) and vastus lateralis (VL) were analyzed and compared. Indeed, it has been suggested that the quadriceps are more affected than the hamstrings in other models such as disuse and in adult aging, ${ }^{17,18}$ and thus an understanding of the entire thigh would highlight the possible differential effects of CIDP on extensor and flexor compartment muscles that might help explain functional variabilities between anterior and posterior thigh muscles. Therefore, the purpose of this study was to assess and compare muscle quantity, as defined by relative muscle density and homogeneity of muscles within different major muscle compartments. Both proximal and distal lower limb segments were compared; specifically the posterior thigh and the posterior leg compartments, respectively, in patients with CIDP and control subjects using T-1 weighted MRI. Additionally, the quadriceps femoris muscles were assessed to provide a comprehensive understanding of the effects of CIDP on all main compartments of the lower limb. Based on the length dependent nature of this disease, it was hypothesized that the triceps surae would have more muscle loss and greater infiltration of non-contractile tissue compared to the hamstrings in CIDP. Furthermore, both the medial hamstrings and quadriceps femoris would have less contractile tissue quantity in the CIDP group compared with matched controls. Moreover, we expected to find greater reduction in muscle quantity in the quadriceps compared to hamstrings due to greater susceptibility of the quadriceps to muscle atrophy as found in other models. ${ }^{17,18}$

\section{Materials and Methods}

\section{Participants}

Five participants with CIDP (four males, one female), mean age $58 \pm 5$ years, and seven age-matched control subjects (five males, two females), mean age $54 \pm 7$ years, were recruited to participate in this study (Table 1). History, clinical, laboratory and electrophysiological features verifying a diagnosis of CIDP were obtained by an experienced neurologist, with specialty training in neuromuscular disorders and electrodiagnosis. The 5 participants with CIDP were a sample of convenience from a neurologist's practice and who were able to volunteer for the MRI procedures. Additionally, other causes of nerve dysfunction (i.e., radiculopathies, other polyneuropathies, or compressive mononeuropathies) were excluded. All CIDP patients were diagnosed based on the criteria established by the European Federation of Neurological Societies (Table 2).$^{12}$ Standard measures of nerve conduction velocities, compound muscle action potential amplitudes (CMAP) (Table 1) and sensory nerve action potential amplitudes were compared to normative values to help confirm the diagnosis of CIDP. Patients with any metabolic (including diabetes), neurological, or vascular diseases (other than those related to CIDP) were not included in this study. Furthermore, all CIDP patients received usual medical care and responded to treatment with either plasma exchange, intravenous immunoglobulin (IVIG), or oral

Table 1. Participant characteristics.

Participant characteristics

Males / Females

Age (years)

Height (m)

Weight (kg)
Controls (n= 7) $5 / 2$

$58 \pm 5.9$

$1.7 \pm 0.1$

$71 \pm 11.2$
CIDP patients $(n=5)$

$4 / 1$

$54.4 \pm 7.7$

$1.7 \pm 0.1$

$77 \pm 13.6$

Values are expressed as means \pm standard deviations. 
Table 2. CIDP clinical classification.

\begin{tabular}{|c|c|c|c|c|c|c|}
\hline Patient & $\begin{array}{c}\text { Duration } \\
\text { of CIDP } \\
\text { (yrs) }\end{array}$ & $\begin{array}{c}\text { CSF level } \\
\text { (g/L isolation) }\end{array}$ & CB & TD & $\begin{array}{c}\text { F-Wave } \\
\text { prolongation/ } \\
\text { latency }\end{array}$ & IVIG or Prednisone Treatment \\
\hline 1 & 22 & 1.5 & $\sqrt{ }$ & $\sqrt{ }$ & - & 80g/ q 4 weeks IVIG \\
\hline 2 & 5 & 0.85 & $\sqrt{ }$ & $\sqrt{ }$ & - & 55g/ q 3 weeks IVIG \\
\hline 3 & 20 & - & $\sqrt{ }$ & $\sqrt{ }$ & $\sqrt{ }$ & 20 mg prednisone daily \\
\hline 4 & 10 & 2.9 & $\sqrt{ }$ & $\sqrt{ }$ & $\sqrt{ }$ & 5mg prednisone every other day \\
\hline 5 & 12 & 6.6 & $\sqrt{ }$ & $\sqrt{ }$ & $\sqrt{ }$ & 55g/ q 4 weeks IVIG \\
\hline
\end{tabular}

prednisone (Table 2). All control subjects were healthy, living independently, medication free and were screened by a neurologist to eliminate any indication of neuromuscular or metabolic disease. The local university research ethics board approved the study, and both groups provided informed oral and written consent prior to testing.

\section{MRI Measures}

Thigh MRI scans were acquired via serial axial plane scans in a 3.0-Tesla magnet (Magnetom Spectra 3T mMRBiograph; Siemens Healthcare, Erlangen, Germany). All MRI scans were completed during a single visit to the magnetic resonance imaging unit. Both CIDP and control subjects were inserted supine into the magnet, feet first, with the thigh iso-centered to the bore of the magnet. Inelastic fastenings were used to firmly secure the thighs, legs, and feet to the MRI table to prevent subtle movements throughout the scan. The complete musculature of the left and right thighs was imaged from the level of the anterior iliac spine to the tibial plateau. MRI for anatomical measures were acquired by means of a 3D FLASH sequence with the following parameters: 9.57-ms repetition time (TR); 2.46-ms echo time (TE); 320 x 240 matrix; 243 x 325mm field of view; 384 slices; and $0.9 \mathrm{~mm}$ slice thickness, with slice separation of $1 \mathrm{~mm}$. Total scan acquisition time for the anatomical scan was approximately 15 min per subject.

\section{Hamstrings and Quadriceps Total Volume and Muscle Composition}

Image processing software (OsiriX version 9.1) was used offline for manual and semi-automated image analysis to measure muscle areas, volumes, and compositions (muscle contractile versus non-contractile tissues) of the medial hamstrings (semitendinosus and semimembranosus) and the quadriceps femoris (vastus lateralis, vastus medialis, rectus femoris, and vastus intermedius). Non-contractile tissue within a muscle compartment were regions around and within muscle tissue that were of obvious different density than muscle and considered to represent connective and adipose tissues. Anatomical determination of the biceps femoris, with its two heads, from the scans was less clear in several participants; therefore, we elected only to assess the medial group that was consistently evident in all scans for volume calculations and in all participants. Inter-rater reliability measures were completed between two experimenters in calculating total muscle volume as well as quantifying contractile versus non-contractile tissue volumes in the hamstrings and quadriceps. Results indicated that both evaluators were within 95\% agreement with each other. The investigator carrying out the analysis was blinded to participant group allocation. Total muscle volumes of the aforementioned muscles were calculated by independently manually delineating regions of interest (ROIs) around every second slice starting from the greater trochanter (proximally) to the tibial plateau (distally). Connective tissue septae, adipose tissue and blood vessels were eliminated during manual tracing of each separate muscle. Slices not outlined manually were interpolated using the "generate missing ROI” function in OsiriX. The investigator then corrected for any computer-generated errors in ROI interpolation. All ROIs within the same muscle series were then saved for further analysis and calculation of non-contractile tissue volumes. The "compute volume" function was used to obtain total volume of each muscle separately.

To calculate non-contractile tissue volumes, an ROI was created for each muscle with only muscle tissue highlighted. Total ROI for each muscle was imported separately and the pixels outside the ROIs were set to zero. A seed point within the muscle being analyzed was selected with a threshold interval being applied using a "grow region tool" to all slices to exclude intramuscular fat, blood vessel, and connective tissue. The investigator then manually corrected for any computer-generated 


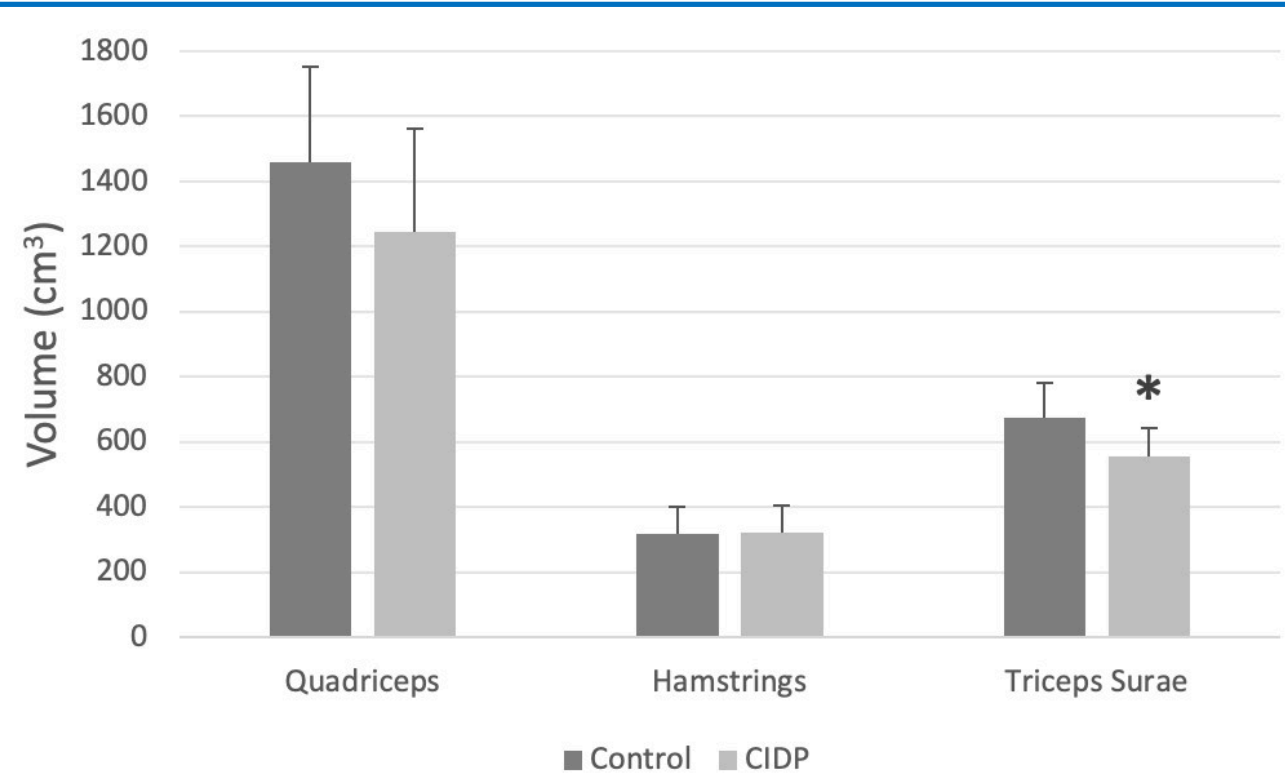

Fig 1. Total compartment volumes (cm3) of the quadriceps (VI, RF, VL, VM), hamstrings (SM, ST) and triceps surae (MG, LG, Sol). *denotes significant difference $(p<0.05)$ between control and CIDP participants. Values are expressed as mean \pm standard deviation.

errors in interpolation. The "compute volume” function was then used to calculate total contractile tissue volume. Non-contractile volume was then calculated by subtracting total contractile tissue from the respective total muscle volume. The procedures used here were identical to those applied to a subset from the prior study for the triceps surae. ${ }^{10}$

\section{Statistics}

Statistical analyses were performed in R (version 3.6). Distribution of data was assessed using the Shapiro-Wilk test of normality. Normally distributed data were analysed using an independent-samples t-test for between group comparison and dependent-sample t-tests were done for within group comparison. Effect sizes were calculated using Cohen's D for within group comparisons of similar sample size. Hedges g was used to calculate effect size between groups where there was not an equal sample size.

\section{Results}

Participant characteristics are presented in Table 1. There were no differences in age, height, and weight between participants with CIDP and control participants. Clinical features of the CIDP group are listed in Table 2.

There was no difference in total quadriceps compartment volume (RF, VI, VM, VL) between CIDP and controls (Figure 1, p>0.05). When intramuscular fat and other non-contractile tissue were removed from the quadriceps CIDP had significantly less (11.5\%) contractile tissue than healthy controls (Figure 2, $\mathrm{p}<0.05$; $\mathrm{g}=2.2$ ). Moreover, within the control group there was significantly less contractile tissue in the quadriceps muscles (total contractile volume $=89 \%$ ) compared to the hamstrings (total contractile volume $=95 \%$ ) (Figure 2 ; $<$ 0.05)

Similarly, total compartment volume for the hamstrings (SM, ST) was not different between controls and CIDP participants (Figure 1, p>0.05), but when non-contractile tissue was removed within the hamstrings, CIDP had significantly less (15.6\%) contractile tissue than controls (Figure 2, $\mathrm{p}<0.05 ; \mathrm{g}=2.8$ ).

With the specific subset of data obtained from Gilmore et al. 2020, we have confirmed that for these same participants, the total compartment volume of the triceps surae (MG, LG, Sol) was significantly lower (17\%) in CIDP than control participants (Figure 1, $\mathrm{p}<0.05$; $\mathrm{g}=1.2$ ). Additionally, CIDP had significantly less (35.9\%) contractile tissue than control participants (Figure 2, $\mathrm{p}<0.05 ; \mathrm{g}=2.5$ ). In comparing different muscles across the same CIDP group of participants we found that the lower amount of contractile tissue in CIDP was significantly greater $(18.7 \%)$ in the triceps surae compared to the hamstrings which are innervated by the same nerve (Figure 2; $\mathrm{p}<0.05 ; \mathrm{D}=1.1$ ).

Figure 3 shows cross-sectional T1-weighted MRI scans comparing the thigh and leg musculature of a typical control with a patient with CIDP; the medial hamstrings (SM and ST), quadriceps (RF, VI, VM, and VL), triceps surae (Sol, MG, and LG), and TA are labelled. Qualitatively, throughout the lower limb the subcutaneous fat layer was more prominent in CIDP compared with controls.

\section{Discussion}

We investigated the possible length dependent effect on quantity of muscle tissue in people with CIDP by focusing on the posterior compartments of the lower 


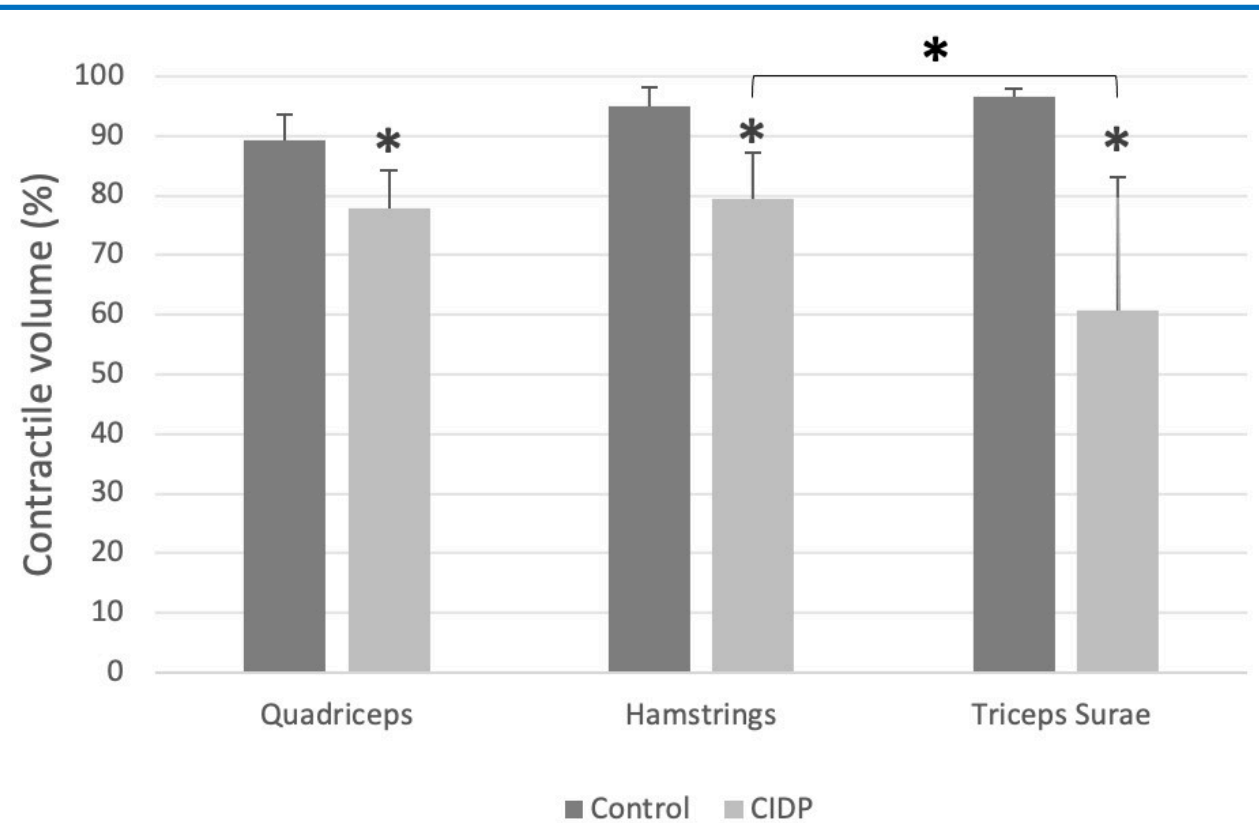

Fig 2. Contractile tissue volume expressed as a \% of total compartment volume in the quadriceps (VI, RF, VL, VM), hamstrings (SM, ST) and triceps surae (MG, LG, Sol). * denotes significant difference $(p<0.05)$. Values are expressed as mean \pm standard deviation

limb; namely the semimembranosus (SM) and semitendinosus (ST) of the hamstrings, with a subset of data in the same participants from a prior study on the triceps surae including soleus (Sol), medial gastrocnemius (MG), and lateral gastrocnemius (LG). Additionally, we assessed the quadriceps femoris muscles: rectus femoris (RF), vastus intermedius (VI), vastus medialis (VM) and vastus lateralis (VL), to provide a comprehensive comparison of anterior and posterior thigh musculature in CIDP and control participants. We found structural alterations within all muscle compartments, showing that those with CIDP have significantly less muscle mass and greater infiltration of non-contractile tissue. Importantly we showed that the triceps surae is significantly more affected than the hamstrings, supporting the premise that CIDP is a length dependent neuropathy that may be reflected by differential changes among muscles innervated by the same nerve axons (sciatic nerve).

We analyzed the proximal extensor and flexor thigh muscles and compared the flexor or hamstring results to those of the triceps surae derived from a subset of participants from a prior study by our group. ${ }^{10}$ The subset (5 CIDP and 7 control participants) was representative of that study (10 CIDP and 9 controls) in that they followed the mean difference with a $\sim 36 \%$ lower amount of contractile tissue volume within CIDP compared to controls in the triceps surae. Whereas in the hamstrings, these same participants within the CIDP group had $~ 16 \%$ less contractile muscle volume than controls. This shows that the triceps surae was significantly more affected than the hamstrings in CIDP, with $\sim 19 \%$ greater loss of contractile tissue in the triceps surae. Our results are supported by Fisse et al. (2020) $)^{14}$ who showed that alterations in ultrasonographic echointensity in CIDP were more prominent in the distal leg muscles compared to proximal, reflecting an increase in fibrosis and fatty infiltration, however this study did not follow muscles innervated by the same nerve. Furthermore, work by Ohyama et al. (2014) ${ }^{13}$ also showed semi-qualitatively using computed tomography that there was significantly more atrophy in the distal musculature compared to proximal in patients with CIDP. We provide a more comprehensive analysis of the total muscle volume by multiple slice reconstruction of the entire segment using MRI to investigate length dependence by assessing muscles innervated by the same (sciatic) nerve. Moreover, strength assessment and electrophysiological studies on patients with CIDP have shown that weakness as well as a reduction in compound muscle action potential amplitudes are more prominent distally within the lower limb. ${ }^{11}$

With a comprehensive analysis of the anterior and posterior thigh, we found that the quadriceps and hamstrings were not different from each other in this group, but rather similarly affected by CIDP. This is an important finding because the quadriceps compared to other muscle groups are significantly more affected with ageing, ${ }^{19,20}$ but this difference was not shown with CIDP, highlighting the impact this disease has on the posterior thigh compartment muscles as well as anterior thigh. Furthermore, the soleus has been shown to be preserved with adult ageing, ${ }^{21}$ however in subjects with CIDP the entire triceps surae showed greater degradation in muscle composition than the hamstrings. This together with the findings from the quadriceps supports that, although 

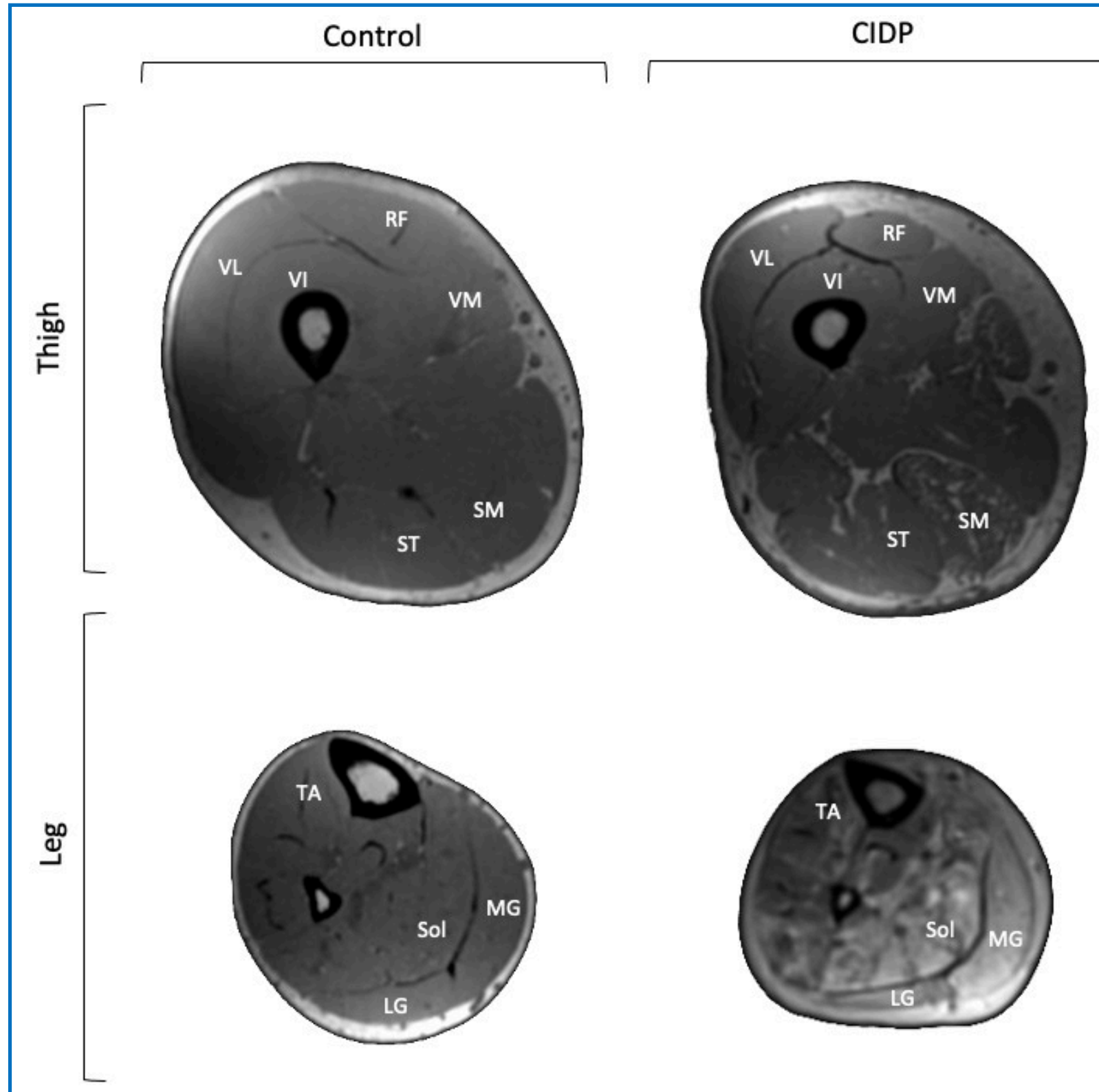

Fig 3. T1-weighted MRI scans comparing cross-section of the mid-thigh and mid-leg musculature of control vs. CIDP. Note the extensive marbleized appearance of the leg in the patient with CIDP, compared to the less thigh musculature. Quadriceps: rectus femoris (RF), vastus intermedius (VI), vastus medialis (VM) and vastus lateralis (VL). Hamstrings: semimembranosus (SM) and semitendinosus (ST). Triceps surae: soleus (Sol), medial gastrocnemius (MG), lateral gastrocnemius (LG). Tibialis anterior (TA).

ageing and disuse factors may play a secondary role in muscle atrophy in middle-aged and older adults, the primary cause in CIDP is likely due to factors related to either inflammatory demyelination, axonal degeneration, or both. ${ }^{10,23}$ Work comparing the sacral plexus (sciatic nerve) innervating the posterior compartment, with the lumbar plexus (femoral nerve) innervating the anterior compartment of the thigh, would provide insight into whether the lack of difference between the quadriceps and hamstrings in CIDP is related to the lumbar plexus as both nerves (femoral and sciatic) share some similar vertebral roots (L4-5). Additionally, in future studies the degree of muscle inflammation per se in the various limb compartments could be evaluated by using other MRI sequences such as short T1 inversion recovery MRI (STIR), ${ }^{24}$ and possible differential affects of treatment strategies (e.g., corticosteroid vs IVIG) on muscle hypertrophy evaluated in larger cohorts. Furthermore, other imaging modalities such as color computed tomography could add value for fine quantitative 
analyzes of skeletal muscle tissue, as previously established in chronic post-traumatic denervation of the thigh and leg muscles. ${ }^{25-27}$ In a previous study in the tibialis anterior (TA), there was no difference in muscle quantity or quality between the posterior leg compartment (i.e. the triceps surae) and the anterior leg compartment (i.e. the TA) within the CIDP group with both showing $~ 50 \%$ less contractile muscle volume than controls.

These findings may indicate that both the tibial and deep fibular nerves, which are distal portions of the sciatic, are affected to similar degrees in CIDP..$^{9,10}$ Although it has been suggested that nerve damage occurs more proximal in CIDP such as with sacral plexus root enlargement, ${ }^{22}$ our results taken together with previous findings suggest that nerve insults may be more distal. Nerve root enlargement is a reflection of the demyelination occurring proximally, but perhaps distally the nerves can become severely demyelinated affecting the fidelity of connections with muscle fibres. This could be due to the greater insults noted at the distal nerve terminals where the blood nerve barrier is anatomically deficient, compared to the more proximal nerve roots. ${ }^{15,16}$

Here we provide a comprehensive anatomical summary into differences in thigh muscle composition, and the length dependent nature of CIDP in which a greater extent of atrophy was found in the distally innervated muscles of the lower limb than more proximal muscles. A better characterization of changes in nerve size along the length of the sciatic nerve would provide additional support to a length dependent effect in CIDP.

\section{List of acronyms}

CIDP - chronic inflammatory demyelinating polyneuropathy

CMAP - compound muscle action potential amplitudes

IVIG - intravenous immunoglobulin

LG - lateral gastrocnemius

MG - medial gastrocnemius

MRI - magnetic resonance imaging

$\mathrm{RF}$ - rectus femoris

ROI - region of interest

$\mathrm{SM}$ - semimembranosus

Sol - soleus

ST - semitendinosus

TA - tibialis anterior

VI - vastus intermedius

$\mathrm{VL}$ - vastus lateralis

$\mathrm{VM}$ - vastus medialis

\section{Authors' contributions}

All authors contributed to the development of the study and framing the question. TJD supported, advised, and helped KJG recruit patients. KJG managed the MR imaging data collection and advised on data analyses. JF and AMZ led primary and secondary data analysis and all authors contributed to interpretation. JF led the writing of the manuscript with contributions from AMZ, and all authors contributed to the revisions and preparation of the final document.

\section{Acknowledgments}

We thank Dr. Kurt Kimpinski MD, PhD for his assistance with patient recruitment and neurological assessments. A special thank you to all participants for volunteering their time and interest.

\section{Funding}

The study was supported by NSERC to C. L. Rice.

\section{Conflict of Interest}

The authors have no conflicts of interest to report.

\section{Ethical Publication Statement}

We confirm that we have read the Journal's position on issues involved in ethical publication and affirm that this report is consistent with those guidelines.

\section{Corresponding Author}

Dr. Charles L. Rice, Present address:

Arthur \& Sonia Labatt Health Sciences Building,

Room 411. London, Ontario, Canada, N6A 5B9

Tel: 519-661-2111 x88918

ORCID iD: 0000-0002-5323-6979

E-mail: crice@uwo.ca

E-mails and ORCID iD of co-authors

Jacob Fanous-jfanous3@uwo.ca

ORCID iD: 0000-0002-5925-4879

Alexander M. Zero-azero@uwo.ca

ORCID iD: 0000-0001-7962-4147

Kevin J. Gilmore - kevin.gilmore@utoronto.ca

ORCID iD: 0000-0002-1126-0890

Timothy J.Doherty - tim.doherty@lhsc.on.ca

ORCID iD: 0000-0002-5883-3975

\section{References}

1. Bromberg $\mathrm{M}$. What is in the literature. J Clin Neuromuscul Dis. 2021 Jun 1;22(4):200-208. doi: 10.1097/CND.0000000000000371.

2. Bosboom WMJ, van den Berg LH, Franssen H, Giesbergen PCLM, Flach HZ, van Putten AM, Veldman H, Wokke HJ. Diagnostic value of sural nerve demyelination in chronic inflammatory demyelinating polyneuropathy. Brain. 2001 Dec;124(Pt 12):2427-38. doi: 10.1093/brain/124. 12.2427

3. Piccolino M. Luigi Galvani's path to animal electricity. J Hist Neurosci. 2008;17(3):335-48. doi: 10.1080/09647040701420198.

4. Seyfarth EA. Julius Bernstein (1839-1917): pioneer neurobiologist and biophysicist. Biol Cybern. 2006 Jan;94(1):2-8. doi: 10.1007/s00422-005-0031-y.

5. Shah S, Morrow JM, Sinclair CDJ, Reilly MM, Thornton JS, Lunn MP, Yousry TA. MRI quantifies lumbosacral nerve root and sciatic nerve hypertrophy in chronic inflammatory 
demyelinating polyradiculoneuropathy. Eur J Radiol. 2020 Sep;130:109164. doi: 10.1016/j.ejrad.2020.109164.

6. Pitarokoili K, Schlamann M, Kerasnoudis A, Gold R, Yoon M-S. Comparison of clinical, electrophysiological, sonographic and MRI features in CIDP. J Neurol Sci. 2015 Oct 15;357(1-2):198203. doi: 10.1016/j.jns.2015.07.030

7. Ishikawa $\mathrm{T}$, Asakura $\mathrm{K}$, Mizutani $\mathrm{Y}$, Ueda A, Murate K-I, Hikichi C, et al. Magnetic resonance neurography for the evaluation of CIDP. Muscle Nerve. 2017 Apr;55(4):483-489. doi: 10.1002/mus.25368.

8. Sinclair CDJ, Morrow JM, Miranda MA, Davagnanam I, Cowley PC, Mehta H, Hanna M, Koltzenburg M, Yousry T, Reilly M, Thornton JS. Skeletal muscle MRI magnetisation transfer ratio reflects clinical severity in peripheral neuropathies. J Neurol Neurosurg Psychiatry. 2012 Jan;83(1):2932. doi: 10.1136/jnnp.2011.246116.

9. Gilmore KJ, Doherty TJ, Kimpinski K, Rice CL. Reductions in muscle quality and quantity in chronic inflammatory demyelinating polyneuropathy patients assessed by magnetic resonance imaging. Muscle Nerve. 2018 Sep;58(3):396-401. doi: 10.1002/mus.26159.

10. Gilmore KJ, Fanous J, Doherty TJ, Kimpinski K, Rice CL. Nerve dysfunction leads to muscle morphological abnormalities in chronic inflammatory demyelinating polyneuropathy assessed by MRI. Clin Anat. 2020 Jan;33(1):77-84 doi: 10.1002/ca.23473.

11. Harbo T, Andersen H, Jakobsen J. Lengthdependent weakness and electrophysiological signs of secondary axonal loss in chronic inflammatory demyelinating polyradiculoneuropathy. Muscle Nerve. 2008 Aug;38(2):1036-45. doi: 10.1002/mus.21000.

12. Bergh FPYK Van Den, Hadden RDM, Bouche P. European Federation of Neurological Societies / Peripheral Nerve Society Guideline on management of chronic inflammatory demyelinating polyradiculoneuropathy: Report of a joint task force of the European Federation of Neurological Societies and the Peripheral Nerve Society - First Revision. Eur J Neurol. 2010 Mar;17(3):356-63. doi: 10.1111/j.1468-1331.2009.02930.x.

13. Ohyama K, Koike H, Katsuno M, Takahashi M, Hashimoto R, Kawagashira Y, et al. Muscle atrophy in chronic inflammatory demyelinating polyneuropathy: A computed tomography assessment. Eur J Neurol. 2014 Jul;21(7):1002-10. doi: 10.1111/ene.12426.

14. Fisse AL, Fiegert S, Stoykova Z, Brunger J, Athanasopoulos D, Gruter T, Motte J, Gold R, Pitarokoili K. Increased muscle echointensity correlates with clinical disability and muscle strength in chronic inflammatory demyelinating polyneuropathy. Eur J Neurol. 2021 May;28(5):1698-1705. doi: 10.1111/ene.14716.

15. Kuwabara S, Ogawara K, Misawa S, Mori M, Hattori T. Distribution patterns of demyelination correlate with clinical profiles in chronic inflammatory demyelinating polyneuropathy. J Neurol Neurosurg Psychiatry. 2002 Jan;72(1):3742. doi: 10.1136/jnnp.72.1.37.

16. Kuwabara S, Misawa S. Chronic inflammatory demyelinating polyneuropathy: Clinical subtypes and their correlation with electrophysiology. Clinical \& Experimental Neuroimmunology. 2011;2:41-48. doi.org/10.1111/j.1759-1961.2011. 00020.x

17. Abe T, Loenneke JP, Thiebaud RS, Loftin M. Agerelated muscle loss of the anterior and posterior thigh assessed by means of MRI/CT and ultrasound. Journal of Trainology. 2014; 3:47-52.

18. Kilroe SP, Fulford J, Jackman SR, Vanloon LJC, Wall BT. Temporal muscle-specific disuse atrophy during one week of leg immobilization. Med Sci Sports Exerc. 2020 Apr;52(4):944-954. doi: 10.1249/MSS.0000000000002200.

19. Maden-Wilkinson T, Degens H, Jones DA, McPhee JS. Comparison of MRI and DXA to measure muscle size and age-related atrophy in thigh muscles. J Musculoskelet Neuronal Interact. 2013 Sep;13(3):320-8.

20. Hogrel JY, Barnouin Y, Azzabou N, ButlerBrowne G, Voit T, Moraux A, Leroux G, Behin A, McPhee JS, Carlier PG. NMR imaging estimates of muscle volume and intramuscular fat infiltration in the thigh: variations with muscle, gender, and age. Age (Dordr). 2015 Jun;37(3):9798. doi: 10.1007/s11357-015-9798-5.

21. Morse CI, Thom JM, Reeves ND, Birch KM, Narici MV. In vivo physiological cross-sectional area and specific force are reduced in the gastrocnemius of elderly men. J Appl Physiol (1985). 2005 Sep;99(3):1050-5.

10.1152/japplphysiol.01186.2004.

22. Ginsberg L, Platts AD, Thomas PK. Chronic inflammatory demyelinating polyneuropathy mimicking a lumbar spinal stenosis syndrome. J Neurol Neurosurg Psychiatry. 1995 Aug;59(2):18991. doi: 10.1136/jnnp.59.2.189.

23. Piccione EA, Engelstad J, Dyck PJ, Mauremann ML, Dispenzieri A, Dyck JB. Nerve pathologic features differentiate POEMS syndrome from CIDP. Acta Neuropathol Commun. 2016 Oct 31;4(1):116. doi: 10.1186/s40478-016-0389-1.

24. Dahlqvist JR, Salim R, Thomsen C, Vissing J. A quantitative method to assess muscle edema using short T1 inversion recovery MRI. Sci Rep. 2020 Apr 29;10(1):7246. doi: 10.1038/s41598-02064287-8.

25. Edmunds KJ, Okonkwo OC, Sigurdsson S, Lose SR, Gudnason V, Carraro U, Gargiulo P. Soft tissue 
radiodensity parameters mediate the relationship between self-reported physical activity and lower extremity function in AGES-Reykjavík participants. Sci Rep. 2021 Oct 11;11(1):20173. doi: 10.1038/s41598-021-99699-7.

26. Ricciardi C, Edmunds KJ, Recenti M, Sigurdsson S, Gudnason V, Carraro U, Gargiulo P. Assessing cardiovascular risks from a mid-thigh CT image: a tree-based machine learning approach using radiodensitometric distributions. Sci Rep. $2020 \mathrm{Feb}$ 18;10(1):2863. doi: 10.1038/s41598-020-59873-9.

27. Recenti M, Ricciardi C, Edmunds K, Gislason MK, Gargiulo P. Machine learning predictive system based upon radiodensitometric distributions from mid-thigh CT images. Eur J Transl Myol. 2020 Apr 1;30(1):8892. doi: 10.4081/ejtm.2019.8892. eCollection 2020 Apr 7.

Submission: October 12, 2021 Revision submitted: November 9, 2021 Accepted for publication: November 112021 